\title{
Vigabatrina aumenta atividade da superóxido dismutase no corpo estriado de ratos após crises convulsivas induzidas pela pilocarpina
}

\author{
Vigabratine increases superoxide dismutase activity in striatum of rat after pilocarpine-induced seizures
}

\author{
RIVELILSOn MENDEs de Freitas ${ }^{1}$ \\ 'Professor adjunto do Setor de Farmacologia da Universidade Federal do Piauí (UFPI), Teresina, PI.
}

Recebido: 2/4/2009 - Aceito: 28/5/2009

\section{Resumo}

Contexto: Tem sido sugerido que durante as crises convulsivas induzidas pela pilocarpina pode ser observado aumento no estresse oxidativo cerebral. Estudos sugerem que compostos com atividade antioxidante podem fornecer certo grau de proteção contra a neurotoxicidade induzida pelas crises convulsivas. Objetivos: $\mathrm{O}$ presente estudo investigou as ações farmacológicas da vigabatrina nas alterações comportamentais e na atividade enzimática da superóxido dismutase (SOD) no corpo estriado de ratos adultos. Métodos: Ratos Wistar adultos (2 meses de idade) foram usados nos experimentos e divididos em quatro grupos. O primeiro foi tratado com solução salina $0,9 \%$ (grupo controle). O segundo grupo foi tratado com pilocarpina ( $400 \mathrm{mg} / \mathrm{kg}$, i.p., grupo P400). O terceiro grupo foi tratado com vigabatrina ( $500 \mathrm{mg} / \mathrm{kg}$, i.p., grupo VGB) e o quarto grupo foi tratado com vigabatrina ( $500 \mathrm{mg} / \mathrm{kg}$, i.p.) e 30 minutos depois com pilocarpina ( $400 \mathrm{mg} / \mathrm{kg}$, i.p., grupo VGB + P400). Os animais que apresentaram crises convulsivas, estado de mal epiléptico e não morreram durante o período de 24 horas de observação foram sacrificados para dissecação do corpo estriado para realização da determinação da atividade da SOD. Resultados: Os estudos comportamentais revelaram que, após administração de pilocarpina, todos os animais apresentaram sinais colinérgicos periféricos, movimentos estereotipados e tremores. No mesmo grupo, foram observados, em $75 \%$ dos animais, crises convulsivas e o estado de mal epiléptico. Por sua vez, o pré-tratamento com vigabatrina produziu redução significativa de $50 \%$ nas crises convulsivas. Com relação aos estudos neuroquímicos, não foram observadas alterações na atividade da SOD no corpo estriado do grupo P400, em comparação aos valores do grupo controle. No entanto, no grupo VGB + P400 foi visto aumento significativo na atividade da SOD de $34 \%$ e 35\%, quando comparado aos grupos controle e P400, respectivamente. Conclusões: Os resultados do presente estudo indicam que durante as crises convulsivas ocorrem alterações comportamentais, entretanto, não foram verificadas mudanças na atividade da SOD durante a fase aguda dessas crises. Esses dados sugerem que os efeitos anticonvulsivantes da vigabatrina podem ser decorrentes da neuromodulação da SOD. No entanto, serão realizados novos estudos neurofarmacológicos para o esclarecimento do mecanismo de ação da vigabatrina no modelo de epilepsia induzido pela pilocarpina.

Freitas RM / Rev Psiq Clín. 2010;37(1):36-40

Palavras-chave: Corpo estriado, crises convulsivas, pilocarpina, superóxido dismutase, vigabatrina.

\begin{abstract}
Background: Pilocarpine-induced seizures have been suggested to be mediated by increases in oxidative stress. Current studies have suggested that antioxidant compounds may afford some level of neuroprotection against the neurotoxicity of seizures. Objectives: This study investigated the pharmacological actions of vigabatrin on behavioral changes and superoxide dismutase (SOD) activity in striatum of adult rats. Methods: Adult rats ( 2 months old) were used in the experiments and divided into four groups. The first was treated with $0.9 \%$ saline (control group). The second group was treated with pilocarpine ( $400 \mathrm{mg} / \mathrm{kg}$, i.p., P400 group). The third group received vigabatrin alone $(500 \mathrm{mg} / \mathrm{kg}$, i.p., VGB group) and the fourth group was treated with vigabatrin (500 $\mathrm{mg} / \mathrm{kg}$, i.p.) and 30 minutes later received pilocarpine (400 mg/kg, i.p., VGB + P400 group). The animals which had seizures and status epilepticus (SE) and did not die within 24 hours of observation were sacrificed to perform the neurochemical studies. Results: Behavioral studies showed that the administration of pilocarpine produces peripheral cholinergic signs, tremors and stereotyped movements in all animals. An amount of $75 \%$ of those rats developed to seizures and SE. In turn, the pre-treatment with vigabatrin produced a 50\% reduction in the rate of seizures and SE. Regarding the neurochemical studies, there were no changes in the striatal SOD activity in P400 group as compared to the control group. However, in the VGB + P400 group it was verified significant increases in SOD activity of $34 \%$ and $35 \%$ as compared to control and P400 group, respectively. Discussion: Our results indicate that behavioral changes occur during seizures, but SOD activity remained unaltered during the acute phase of the convulsive crisis. Our findings suggest that the anticonvulsant effect of vigabatrin may be the result of modulation of this enzyme, in an attempt to protect the animal against neuronal damage produced by seizures. Further pharmacological studies will be carried out in order to clarify the mechanism of action of vigabatrin on the pilocarpine-induced epilepsy model.
\end{abstract}

Freitas RM / Rev Psiq Clín. 2010;37(1):36-40

Key-words: Striatum, seizures, pilocarpine, superoxide dismutase, vigabatrin.

\section{Introdução}

A epilepsia é uma condição crônica que apresenta diferentes etiologias, tratamentos e prognósticos ${ }^{1}$. As crises convulsivas induzidas pela pilocarpina também estão associadas a várias mudanças neuroquímicas no corpo estriado de ratos $^{2}$. Essas crises convulsivas podem alterar a síntese, a liberação e/ou a biotransformação de vários neurotransmissores, entre eles do GABA e glutamato ${ }^{3}$, como também produzem mudanças na atividade de enzimas antioxidantes ${ }^{4}$.
O modelo de convulsão induzido pela pilocarpina em animais é bastante utilizado para estudar a fisiopatologia das crises convulsivas, uma vez que reproduz as alterações comportamentais e eletroencefalográficas que são semelhantes à epilepsia do lobo temporal de humanos ${ }^{5}$. Esse modelo também é essencial para a caracterização dos mecanismos de ações de drogas anticonvulsivantes para o tratamento da epilepsia e, além disso, pode ser utilizado para estudar o envolvimento dos sistemas antioxidantes como moduladores da epileptogênese ${ }^{6}$. 
Várias alterações na concentração da peroxidação lipídica, no conteúdo do nitrito, no nível da glutationa reduzida e na atividade antioxidante enzimática foram verificadas durante a fase aguda das crises convulsivas ${ }^{4}$. De modo geral, os radicais livres têm sido implicados na patogênese de várias doenças neurológicas, incluindo a epilepsia. Os radicais livres podem produzir várias alterações neuroquímicas no corpo estriado de ratos adultos durante as crises convulsivas $^{2}$ e são produzidos e removidos do organismo por meio de um rigoroso controle pelos sistemas de defesa antioxidante. O cérebro é mais vulnerável que outros tecidos ao estresse oxidativo porque apresenta menos mecanismos de defesa antioxidante e uma grande quantidade de lipídios e metais oxidáveis em relação aos outros tecidos ${ }^{7}$.

Diante do exposto, seria importante verificar a ação de drogas anticonvulsivantes na atividade de enzimas antioxidantes, uma vez que o estresse oxidativo tem uma importante participação na neurogênese das crises convulsivas induzidas pela pilocarpina. A vigabatrina é uma droga antiepiléptica atualmente licenciada em mais de 85 países para o tratamento da epilepsia focal em adultos e para espasmos infantis ${ }^{8-11}$. É útil também para o tratamento de crises convulsivas associadas com a esclerose tuberosa ${ }^{12}$. É um inibidor seletivo e irreversível da enzima GABA-transaminase, que catalisa a inativação do ácido $\gamma$-aminobutírico (GABA). Seu efeito anticonvulsivante é produzido pelo aumento da concentração do $\mathrm{GABA}^{13}$. Estudos sugerem que a elevação dos níveis de GABA no córtex cerebral produzido pela vigabatrina pode reduzir o aumento da dopamina extracelular observado durante as crises convulsivas ${ }^{14}$

Em recente trabalho, foi sugerido que o dano neuronal produzido pelas crises convulsivas induzidas pela pilocarpina pode ser resultante do aumento dos níveis de peroxidação lipídica e nitrito no corpo estriado de ratos após crises convulsivas, no entanto, não foi evidenciado aumento compensatório na atividade da superóxido dismutase nessa área durante essas crises convulsivas ${ }^{2}$.

\section{Objetivos}

Com base nesses pressupostos, o objetivo do presente estudo foi investigar os efeitos anticonvulsivantes e as alterações na atividade da superóxido dismutase no corpo estriado de ratos adultos prétratados com vigabatrina durante a fase aguda das crises convulsivas induzidas pela pilocarpina.

\section{Material e métodos}

Foram utilizados ratos Wistar machos, adultos (2 meses de idade, 250-280 g). Durante todos os experimentos, os animais foram mantidos em gaiolas de acrílico de 30 × $30 \times 30 \mathrm{~cm}^{3}$, onde foram observados durante 24 horas com, no máximo, seis animais, em condições controladas (temperatura ambiente de $24-25^{\circ} \mathrm{C}$ e umidade de $50 \%$ a 60\%), com ciclo claro/escuro alternado de 12 horas (7:00 a.m. a 7:00 p.m.), recebendo ração padrão tipo Purina e água ad libitum. Os experimentos foram realizados de acordo com o guia de cuidados e usos de animais de laboratório do Departamento de Saúde e Serviços Humanos dos Estados Unidos da América (EUA). As doses das drogas usadas foram determinadas a partir de dois estudos de dose-resposta, incluindo pilocarpina (dados não mostrados) e por meio das observações das doses utilizadas atualmente em estudos com modelos animais descritos na literatura ${ }^{6}$. As doses utilizadas não são equivalentes às utilizadas em humanos, porque os ratos têm diferentes taxas metabólicas.

Um grupo de animais foi tratado com pilocarpina ( $400 \mathrm{mg} / \mathrm{kg}$, i.p., $\mathrm{n}=18$, grupo P400) e observado por 24 horas após a administração. Os animais controles foram tratados com solução salina $(\mathrm{NaCl}, 0,9 \%, \mathrm{n}=14$, controle). Um terceiro grupo de animais foi tratado com vigabatrina $(500 \mathrm{mg} / \mathrm{kg}$, i.p., $\mathrm{n}=12)$ e, 30 minutos após esse tratamento, os animais foram tratados com pilocarpina (400 mg/kg, i.p., VGB + P400 grupo). Outro grupo foi tratado somente com vigabatrina $(500 \mathrm{mg} / \mathrm{kg}$, i.p., $\mathrm{n}=12$, VGB). Após 24 horas da administração de pilocarpina, os animais que apresentaram crises convulsivas, estado de mal epiléptico e sobreviveram ao tratamento foram sacrificados sem anestesia por decapitação em guilhotina (Havard, USA) e, em seguida, seus cérebros foram removidos. O corpo estriado foi dissecado sobre gelo e armazenado em papel alumínio devidamente identificado, pesado e conservado a $-80^{\circ} \mathrm{C}$ para uso posterior. Os grupos controle, VGB e VGB + P400 também foram sacrificados da mesma forma. As dosagens neuroquímicas da atividade da superóxido dismutase estriatal foram realizadas imediatamente após a dissecação.

Os animais tratados e controles foram divididos em gaiolas contendo, no máximo, seis animais e colocados em ambiente reservado, sendo feita a observação direta. Todos os grupos experimentais foram observados após os tratamentos, de acordo com os protocolos experimentais, perfazendo um total de 24 horas de observação. Os seguintes parâmetros foram observados: presença de sinais colinérgicos periféricos (miose, piloereção, cromodacriorreia, salivação, diarreia e diurese), tremores, movimentos estereotipados (aumento da atividade de roer, coçar, mastigar e wet-dog shakes (ato de sacudir - semelhante a um cachorro molhado), crises convulsivas (incluindo movimentos clônicos das extremidades superiores que ocorrem em aproximadamente 30 minutos após a administração da pilocarpina), progredindo para o estado de mal epiléptico (caracterizado por crises convulsivas definidas como contínuas quando ocorrem por um período maior que 30 minutos) e ainda se determinou a taxa de mortalidade de cada grupo experimental. Depois desse período de observação, os animais foram sacrificados por decapitação e seus cérebros, removidos para a dissecação do corpo estriado para a realização dos estudos neuroquímicos. O grupo P400 foi constituído pelos ratos que apresentaram crises convulsivas, estado de mal epiléptico e não morreram durante as 24 horas de observação.

A atividade da SOD foi determinada por meio da técnica descrita anteriormente ${ }^{15}$. Foi preparado o meio da reação contendo tampão fosfato de potássio $50 \mathrm{mM}, \mathrm{pH} 7,8$, xantina $500 \mu \mathrm{M}$; cianeto de potássio $200 \mu \mathrm{M}$; EDTA $1 \mathrm{mM}$. Os homogenatos (10\% $\mathrm{p} / \mathrm{v}$ ) foram preparados com tampão fosfato de potássio $50 \mathrm{mM}, \mathrm{pH}$ 7,8 e, em seguida, centrifugados a 15.000 rpm durante 15 minutos a $4^{\circ} \mathrm{C}$. Os sobrenadantes foram removidos para determinação da atividade da SOD estriatal. A xantina oxidase $(\mathrm{XO}-5 \mathrm{U} / \mathrm{mL})$ usada na reação foi preparada a partir da solução padrão de XO $(1 \mu \mathrm{L}$ para $80 \mu \mathrm{L}$ de tampão fosfato de potássio $50 \mathrm{mM}, \mathrm{pH}$ 7,8). Em seguida, foi realizado o ensaio contendo $975 \mu \mathrm{L}$ do meio reacional, $20 \mu \mathrm{L}$ da amostra e $5 \mu \mathrm{L}$ da XO e a mistura foi agitada e, na sequência, realizada a leitura a $550 \mathrm{~nm}$ durante 6 minutos. A leitura do branco cinético foi feita a $550 \mathrm{~nm}$. A quantidade da atividade da SOD das amostras foi calculada usando a média das absorções lineares obtidas durante 6 minutos pela curva. Os resultados foram expressos em U/ mg de proteína. Uma unidade (U) da atividade enzimática da SOD corresponde à inibição de $50 \%$ da reação do $\mathrm{O}_{2}^{-}$com o citocromo C. A concentração da proteína foi obtida pelo método descrito por Lowry et al..$^{16}$.

Para análise estatística dos resultados que obedeciam a uma distribuição paramétrica, foi utilizada a análise de variância (Anova) para múltiplas comparações e o teste de Student Newman Keuls como post hoc test pelo programa GraphPad Prism versão 3.00 para Windows, GraphPad Software, San Diego California USA. Copyright (c) 1994-1999 por GraphPad software. Os dados não paramétricos (percentagens) foram analisados pelo mesmo programa utilizando o teste do qui-quadrado. As diferenças foram consideradas estatisticamente significativas a partir de $\mathrm{p}<0,05$.

\section{Resultados}

Os estudos comportamentais foram realizados como descrito anteriormente. Todos os animais adultos tratados com pilocarpina (400 mg/kg, sc; $\mathrm{n}=18, \mathrm{P} 400)$, durante 24 horas de observação, apresentaram sinais colinérgicos periféricos (SCP), tais como miose, piloereção, cromodacriorreia, salivação, diarreia, diurese, 
e também movimentos estereotipados (ME), envolvendo o aumento da atividade de roer, coçar, mastigar e wet-dog shakes (ato de sacudir - semelhante a um cachorro molhado) e tremores. As crises convulsivas ocorreram em 75\% (14) que apareceram nos 25 minutos iniciais. Em todos os animais que apresentaram crises, $75 \%$ (14), foi verificada a instalação do estado de mal epiléptico. Observou-se um índice de sobrevivência de 40\% (7) no mesmo grupo (Tabela 1).

Tabela 1. Efeitos de pré-tratamento com vigabatrina nas alterações comportamentais em ratos adultos durante as crises convulsivas induzidas pela pilocarpina

\begin{tabular}{l|c|c|c|c|c}
\hline Grupos & $\begin{array}{c}\text { Latência de } \\
\text { instalação da } \\
\text { primeira crise } \\
\text { convulsiva } \\
(\mathrm{min})\end{array}$ & $\begin{array}{c}\text { Crises } \\
\text { convulsivas } \\
(\%)\end{array}$ & $\begin{array}{c}\text { Estado } \\
\text { de mal } \\
\text { epiléptico } \\
(\%)\end{array}$ & $\begin{array}{c}\text { Taxa de } \\
\text { mortalidade } \\
(\%)\end{array}$ & $\begin{array}{c}\text { Número } \\
\text { de } \\
\text { animais }\end{array}$ \\
\hline Controle & 00 & 00 & 00 & 00 & 14 \\
\hline P400 & $35,00 \pm 0,70$ & 75 & 75 & 60 & 18 \\
\hline VGB + P400 & $111,65 \pm 0,45^{\mathrm{c}}$ & $25^{\mathrm{a}}$ & $25^{\mathrm{a}}$ & $20^{\mathrm{a}}$ & 12 \\
\hline VGB & 00 & $00^{\mathrm{a}, \mathrm{b}}$ & $00^{\mathrm{a}, \mathrm{b}}$ & $00^{\mathrm{a}, \mathrm{b}}$ & 12 \\
\hline
\end{tabular}

0 grupo P400 foi tratado com pilocarpina ( $400 \mathrm{mg} / \mathrm{kg}$, i.p., $\mathrm{n}=18, \mathrm{P} 400)$. 0 grupo VGB + P400 foi pré-tratado com vigabatrina ( $500 \mathrm{mg} / \mathrm{kg}$, i.p.) e 30 minutos após recebeu pilocarpina ( $400 \mathrm{mg} / \mathrm{kg}$ i.p., $n=12, V G B+P 400$ ). Os animais controles foram tratados com salina $0,9 \%$ (i.p., $n=14$ ). Outro grupo foi tratado somente com vigabatrina ( $500 \mathrm{mg} / \mathrm{kg}$, i.p., VGB, $\mathrm{n}=12$ ). Após os tratamentos, os animais foram observados durante 24 horas para a determinação da latência para instalação da primeira crise convulsiva, crises convulsivas, estado de mal epiléptico e taxa de mortalidade de cada grupo. Os resultados dos estudos comportamentais foram expressos em percentagem e a latência para instalação da primeira crise convulsiva em minutos (min). a quando comparado ao grupo P400 ( $p<0,05$; teste do qui-quadrado); ${ }^{b}$ quando comparado ao grupo VGB + P400 $\left(p<0,05\right.$; teste do qui-quadrado); ${ }^{c}$ quando comparado ao grupo P400 ( $p<0,05 ;$ Anova e teste de Student Newman Keuls como post hoc teste).

A latência de instalação da primeira crise convulsiva nesse grupo é aproximadamente $35,00 \pm 0,70$ minutos. O pré-tratamento com vigabatrina, 30 minutos antes da administração de pilocarpina, produziu as alterações comportamentais descritas na tabela 1 . Nenhuma alteração nos índices de SCP, ME e tremores observados durante os estudos comportamentais no grupo P400 foi modificada pelo pré-tratamento com vigabatrina. Entretanto, nesse grupo VGB + P400 foi observada redução de forma significativa de 50\% nos índices das crises convulsivas e do estado de mal epiléptico ( $\mathrm{p}<0,0001)$, como também se verificou uma redução na taxa de mortalidade em 40\% ( $p<0,0001)$ em comparação ao grupo P400 (Tabela 1). Com relação à latência de instalação da primeira crise convulsiva, foi observado aumento de $219 \%$ nesse grupo $(111,65$ $\pm 0,45 \mathrm{~min}$ ), quando comparado ao grupo P400 ( $<<0,0001)$. Nenhum dos animais tratados somente com solução salina $0,9 \%$ ou vigabatrina apresentou alterações comportamentais características das crises convulsivas induzidas pela pilocarpina (Tabela 1).

A figura 1 apresenta os efeitos do pré-tratamento com vigabatrina na atividade da SOD no corpo estriado de ratos adultos após crises convulsivas induzidas pela pilocarpina. No grupo P400, não foi verificada nenhuma alteração significativa na atividade da SOD em relação ao grupo controle (controle $=2,38 \pm 0,14 ; \mathrm{P} 400=2,41 \pm 0,18$ ) $(\mathrm{p}<0,8955)$ no corpo estriado dos ratos adultos. Por sua vez, o prétratamento com vigabatrina, 30 minutos antes da administração de pilocarpina, produziu aumento significativo na atividade enzimática da estriatal SOD de $34 \%$ e $35 \%$ quando comparado ao grupo $\mathrm{P} 400$ $(\mathrm{P} 400=2,41 \pm 0,18 ; \mathrm{VGB}+\mathrm{P} 400=3,22 \pm 0,22)(\mathrm{p}<0,0146)$ e ao grupo controle (controle $=2,38 \pm 0,14 ; \mathrm{VGB}+\mathrm{P} 400=3,22 \pm 0,22)$ $(\mathrm{p}<0,0047)$, respectivamente (Figura 1). No grupo tratado somente com vigabatrina, nenhuma alteração significativa foi observada na atividade da SOD no corpo estriado dos ratos adultos em comparação ao grupo controle (controle $=2,38 \pm 0,14 ; \mathrm{VGB}=2,37 \pm 0,12$ ) $(\mathrm{p}<0,9590)$ (Figura 1).

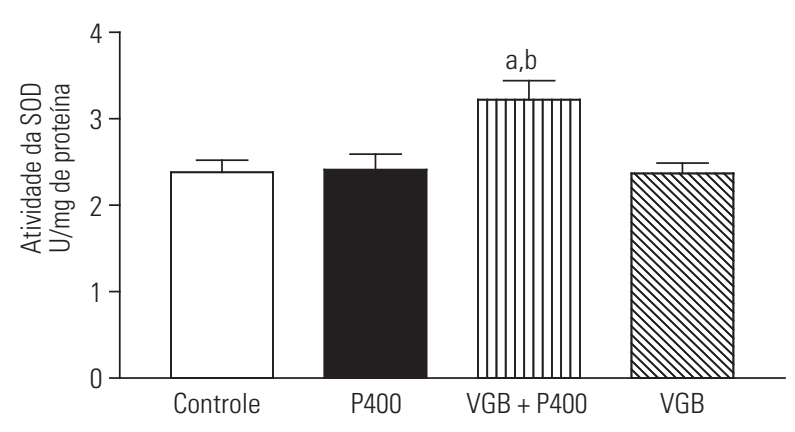

Figura 1. Efeitos da vigabatrina na atividade da superóxido dismutase (SOD) no corpo estriado de ratos adultos após crises convulsivas induzidas pela pilocarpina.

0 grupo P400 foi tratado com pilocarpina (400 mg/kg, i.p., $n=9 ; P 400) .0$ grupo VGB + P400 foi pré-tratado com vigabatrina (500 mg/kg, i.p.) e, 30 minutos após essa administração, recebeu pilocarpina (400 mg/kg, i.p., $n=7$; VGB + P400). Os animais controles foram tratados com salina $0,9 \%$ (i.p., $n=7$ ). Outro grupo foi tratado somente com vigabatrina ( $500 \mathrm{mg} / \mathrm{kg}$, i.p., VGB, $\mathrm{n}=7$ ). Os animais estudados apresentaram crises convulsivas, estado de mal epiléptico e foram sacrificados 24 horas após os tratamentos. Os valores representam a média + EPM do número de animais utilizados em cada grupo. Para análise estatística, foram usados Anova e teste $t$ Student Newman Keuls como post hoc teste. ${ }^{a}$ quando comparado ao grupo controle $(p<0,05)$; ${ }^{\mathrm{b}}$ quando comparado ao grupo $\mathrm{P} 400(\mathrm{p}<0,05)$.

\section{Discussão}

O mecanismo de instalação das crises convulsivas induzidas pela pilocarpina é complexo e diversas vias de neurotransmissão central estão implicadas ${ }^{17-19}$. Manipulações farmacológicas com drogas que agem em diferentes sítios de ação do SNC têm demonstrado a capacidade destas de atenuarem a propagação das crises convulsivas induzidas pela pilocarpina.

O modelo de convulsão induzido pela pilocarpina foi utilizado para investigar as ações anticonvulsivantes da vigabatrina nos parâmetros comportamentais e na atividade da enzima superóxido dismutase no corpo estriado de ratos adultos durante a fase aguda das crises convulsivas. Imediatamente após a administração de pilocarpina nesses experimentos, os ratos apresentaram persistentes alterações comportamentais, incluindo piloereção, cromodacriorreia, acinesia, ataxia, tremores, automatismos mastigatórios como mioclonia dos músculos faciais e movimentos semelhantes a cachorro molhado, que persistiram de 10 a 15 minutos. Essas alterações comportamentais foram contínuas até a instalação das crises convulsivas, incluindo movimentos clônicos das extremidades superiores que ocorreram em 75\% dos animais em aproximadamente 30 minutos após administração da pilocarpina. No mesmo grupo, as crises convulsivas progrediram até o desenvolvimento do estado de mal epiléptico em $75 \%$ destes e ainda foi observada uma taxa de sobrevivência de $40 \%$. Esses achados estão de acordo com os anteriormente descritos na literatura ${ }^{3,20,21}$.

No presente estudo, demonstrou-se que a administração sistêmica de vigabatrina reduz o número de animais que apresentam crises convulsivas e o estado de mal epiléptico, bem como aumenta o índice de sobrevivência. Os resultados sugerem que a vigabatrina pode apresentar uma importante ação anticonvulsivante no modelo de convulsão induzido pela pilocarpina. Entretanto, não foi observada nenhuma mudança nos sinais colinérgicos periféricos, movimentos estereotipados e tremores. Essas alterações podem ser vistas durante as crises convulsivas e são decorrentes da estimulação dos receptores colinérgicos muscarínicos $\mathrm{M}_{3}$. Esses achados sugerem que a ação da vigabatrina não é mediada por meio do bloqueio desses receptores muscarínicos, uma vez que este agente antiepiléptico apresentou importantes efeitos anticonvulsivantes no modelo de epilepsia induzido pela pilocarpina, embora não seja utilizado na clínica para o tratamento da epilepsia do lobo temporal. Assim, mais estudos 
com relação aos outros neurotransmissores envolvidos nas crises convulsivas devem ser realizados para esclarecer o mecanismo de ação anticonvulsivante da vigabatrina nesse modelo de epilepsia.

Alguns estudos sugerem que a hiperexcitabilidade neuronal observada durante as crises convulsivas pode ser produzida pela diminuição da neurotransmissão GABAérgica, pela remoção de radicais livres e pelo aumento da atividade enzimática antioxidante e da $\mathrm{Na}^{+} \mathrm{K}^{+}$ATPase $^{22}$. A alteração no metabolismo oxidativo produz mudanças na síntese e/ou na liberação de monoaminas e aminoácidos que podem estar implicadas na propagação e/ou na manutenção das crises convulsivas ${ }^{23}$. O tratamento com a vigabatrina aumenta a expressão da $\mathrm{Na}^{+} \mathrm{K}^{+} \mathrm{ATPase}^{24}$ e participa da neuromodulação dos autorreceptores $\mathrm{GABA}_{\mathrm{B}}{ }^{25}$. Além dessas modulações cerebrais provocadas na neurotransmissão GABAérgica durante as crises convulsivas, também tem sido sugerido que o mecanismo de ação da vigabatrina pode ser decorrente das mudanças na atividade enzimática antioxidante. Diante disso, decidiu-se investigar os efeitos da vigabatrina nas alterações comportamentais e na atividade antioxidante da superóxido dismutase durante as crises convulsivas induzidas pela pilocarpina.

Têm sido observados, durante as crises convulsivas induzidas pela pilocarpina, diminuição dos receptores GABAérgicos estriatais e aumento na produção de peroxidação lipídica e no conteúdo de nitrito $^{2}$. No entanto, nenhuma mudança na atividade da superóxido dismutase tem sido verificada nessa área. Essa enzima é a principal defesa antioxidante cerebral e o radical livre removido pela sua atividade, o superóxido $\left(\mathrm{O}_{2}^{-}\right)$, está implicado na neuropatogênese das crises convulsivas. A disfunção mitocondrial induzida pela diminuição da atividade da $\mathrm{Na}^{+} \mathrm{K}^{+}$ATPase durante as crises convulsivas pode ser um dos responsáveis pelo aumento da produção desse radical. Esse radical pode ser removido pela ação da superóxido dismutase ${ }^{26}$, portanto, drogas capazes de modular a atividade dessa enzima pode reduzir os efeitos neurotóxicos mediados pelo íon superóxido durante as crises convulsivas.

Investigaram-se os efeitos da vigabatrina na atividade da SOD no corpo estriado de ratos adultos durante a fase aguda das crises convulsivas induzida pela pilocarpina. Não foi verificada mudança significativa na atividade da SOD estriatal durante essas crises. Os dados do presente estudo sugerem que a função antioxidante da superóxido dismutase pode não ser capaz de proteger contra o dano neuronal produzido pelas crises convulsivas. Acredita-se que essa enzima não esteja implicada nos mecanismos fisiopatológicos compensatórios contra a instalação das crises convulsivas ${ }^{27}$. Esses achados sugerem que os radicais livres produzidos nessa região cerebral podem ser essenciais para a propagação e/ou manutenção das crises convulsivas. Por sua vez, o pré-tratamento com a vigabatrina produziu aumento na atividade enzimática da SOD estriatal. Essas mudanças sugerem que, durante as crises, pode não haver envolvimento na atividade da SOD no decorrer da propagação destas. Por outro lado, a vigabatrina foi capaz de reduzir o número de animais que apresentaram crises e que progrediram para o estado de mal epiléptico, bem como aumentou a taxa de sobrevivência, possivelmente devido ao aumento da remoção dos radicais livres superóxidos produzidos durante as crises convulsivas.

Estudos sugerem que a vigabatrina produz seus efeitos anticonvulsivantes por meio da diminuição da atividade da enzima GABA-transaminase $e^{28}$, pelo aumento da liberação do GABA e pela diminuição da concentração da dopamina e taurina cerebral ${ }^{29}$. A vigabatrina pode exercer efeito neuroprotetor contra o dano neuronal produzido pelo aumento no conteúdo de radicais livres durante as crises convulsivas. A literatura sugere que esse efeito pode ser mediado por meio da remoção desses radicais em decorrência do aumento da atividade da $\mathrm{Na}^{+} \mathrm{K}^{+}$ATPase e pela diminuição do processo de oxidação de lipídios e proteínas ${ }^{30}$. Em razão dos efeitos farmacológicos proeminentes durante o estresse oxidativo e na modulação enzimática da SOD verificada nestes experimentos, sugere-se que os efeitos anticonvulsivantes da vigabatrina podem ser decorrentes da modulação da atividade de enzimas antioxidantes cerebrais.
Novos estudos devem ser realizados para uma melhor avaliação e esclarecimento dos efeitos anticonvulsivantes da vigabatrina no modelo de epilepsia induzido pela pilocarpina. Estudos relacionados aos outros sistemas antioxidantes e de neurotransmissão devem ser realizados para esclarecer prontamente os mecanismos implicados nas ações anticonvulsivantes da vigabatrina durante as crises convulsivas induzidas pela pilocarpina.

\section{Referências}

1. De Lorenzo RJ, Churn SB, Kochan LD. Long-term alteration of calcium homeostastic mechanisms in the pilocarpine model of temporal lobe epilepsy. Brain Res. 2001;903:1-12.

2. Freitas RM, Sousa FCF, Vasconcelos SMM, Viana GSB, Fonteles MMF. Pilocarpine-induced seizures in adult rats: lipid peroxidation level, nitrite formation, GABAergic and glutamatergic receptor alterations in the hippocampus, striatum and frontal cortex. Pharmacol Biochem Behav. 2004;78:327-32.

3. Cavalheiro EA, Fernandes MJ, Turski L, Naffah-Mazzacoratti MG. Spontaneous recurrent seizures in rats: amino acid and monoamine determination in the hippocampus. Epilepsia. 1994;35:1-11.

4. Freitas RM, Souza FCF, Vasconcelos SMM, Viana GSB, Fonteles MMF. Oxidative stress in the hippocampus after status epilepticus in rats. FEBS J. 2005;272:1307-12.

5. Ben-Ari Y, Tremblay E, Ottersen OP. Electrographic, clinical and pathological alterations following systemic administration of kainic acid, bicuculine, deoxyglucose or pentylenetetrazole: metabolite mapping using the deoxyglucose method with special reference to the pathology of epilepsy. Neuroscience. 1981;6:1361-91.

6. Pereira MB, Freitas RL, Assis MA, Silva RF, Fonteles MM, Freitas, et al. Study pharmacologic of the GABAergic and glutamatergic drugs on seizures and status epilepticus induced by pilocarpine in adult Wistar rats. Neurosci Lett. 2007;419:253-7.

7. Walz R, Moreira JCF, Benfato MS, Quevedo J, Schorer N, Vianna MMR. Lipid peroxidation in hippocampus early and late after status epilepticus induced by pilocarpine of kainic acid in Wistar rats. Neurosci Lett. 2000;291:179-82.

8. Cramer JA, Fisher R, Ben-Menachem E, Jacqueline F, Mattson RH. New antiepileptic drugs: comparison of key clinical trials. Epilepsia. 1999;40:590-600.

9. Marson AG, Kadir ZA, Hutton JL, Chadwick DW. The new antiepileptic drugs: a systematic review of their efficacy and tolerability. Epilepsia. 1997;38:859-88.

10. Appleton RE, Peters AC, Mumford JP, Shaw DE. Randomised, placebocontrolled study of vigabatrin as first-line treatment of infantile spasms. Epilepsia. 1999;40:1627-33.

11. Lawthom C, Smith PEM, Wild JM. Nasal retinal nerve fiber layer attenuation: a biomarker for vigabatrin toxicity. Ophthalmology. 2009;116:565-71.

12. Marciani MG, Gigli GL, Orlandi L. Long follow-up of tuberous sclerosis treated with vigabatrin. Lancet. 1992;340:1554.

13. Fechtner RD, Khouri AS, Figueroa E, Ramirez M, Federico M, Dewey SL, et al. Short-term treatment of cocaine and/or methamphetamine abuse with vigabatrin: ocular safety pilot results. Arch Ophthalmol. 2006;124:1257-62.

14. Gerasimov MR, Schiffera WK, Gardnerb EL, Marstellera Da, Lennonc IC, Taylorc SJC. GABAergic blockade of cocaine-associated cue-induced increases in nucleus accumbens dopamine. Eur J Pharmacol. 2001;414:205-9.

15. Flohe L, Otting F. Superoxide dismutase assays. Methods Enzymology. 1984;105:93-104.

16. Lowry OH, Rosebrough NJ, Farr AL, Randall RJ. Protein measurement with follin phenol reagent. J Biol Chem. 1951;193:265-75.

17. Brooks-Kayal AR, Shumate MD, Jin H, Lin DD, Rikther TY, Holloway KL, et al. Human neuronal gamma-aminobutyric acid(A) receptors: coordinated subunit mRNA expression and functional correlates in individual dentate granule cells. J Neuroscience. 1999;19:8312-8.

18. Freitas RM, Viana GSB, Fonteles MMF. Níveis dos neurotransmissores estriatais durante o estado epiléptico. Rev Psiq Clín. 2003;30:76-9.

19. Negrão N, Kuczynski E, Thomé-Souza S, Valente KDR. Depressão em crianças e adolescentes com epilepsia. Rev Psiq Clín. 2004;31:290-9. 
20. Marinho MMF, Sousa FCF, Bruin VMS, Vale MR, Viana GSB. Effects of lithium, alone or associated with pilocarpine, on muscarinic and dopaminergic receptors and on phosphoinositide metabolism in rat hippocampus and striatum. Neurochem Int. 1998;33:299-306.

21. Turski WA, Cavalheiro EA, Schwartz M, Czuczwar SJ, Kleinrok Z, Turski L. Limbic seizures produced by pilocarpine in rats: a behavioural, electroencephalographic and neuropathological study. Behav Brain Res. 1983;9:315-35.

22. Vaillend C, Mason SE, Cuttle MF, Alger BE. Mechanisms of neuronal hyperexcitability caused by partial inhibition of $\mathrm{Na}^{+} \mathrm{K}^{+}$ATPases in the rat CA1 hippocampal region. J Neurophysiol. 2002;88:2963-78.

23. Vizi ES, Sperlágh B. Separation of carrier mediated and vesicular release of GABA from rat brain slices. Neurochem Int. 1999;34:407-13.

24. Kang TC, Kim HS, Seo MO, Park SK, Kwon HY, Kang JH, et al. The changes in the expressions of GABA transporters in the gerbil hippocampal complex following spontaneous seizure. Neurosci Lett. 2001;310:29-32.

25. Jacksom MF, Esolin B, Capek R. Reversal of the activity-dependent suppression of GABA-mediated inhibition in hippocampal slices from gamma-vinil GABA (vigabatrin)-pretreated rats. Neuropharmacology. 2000;39:65-74.

26. Naffah-Mazzacoratti MG, Cavalheiro EA, Ferreira EC, Abdalla DSP, Amado D, Bellissimo MI. Superoxide dismutase, glutathione peroxidase activities and the hydroperoxide concentration are modified in the hippocampus of epileptic rats. Epilepsy Res. 2001;46:121-8.

27. Marinho MMF, Sousa FCF, Bruin VMS, Aguiar LMV, Pinho RSN, Viana GSB. Inhibitory action of a calcium channel blocker (nimodipine) on seizures and brain damage induced by pilocarpine and lithiumpilocarpine in rats. Neurosci Lett. 1997;235:13-6.

28. Todd KG, Baker GB. Neurochemical effects of the monoamine oxidase inhibitor phenelzine on brain GABA and alanine: a comparison with vigabatrin. J Pharm Pharm Sci. 2008;11:14s-21s.

29. Jammoul F, Wang Q, Nabbout R, Coriat C, Duboc A, Simonutti M, et al. Taurine deficiency is a cause of vigabatrin-induced retinal phototoxicity. Ann Neurol. 2009;65:98-107.

30. Bist $R, B$ hatt $D K$. The evaluation of effect of alpha-lipoic acid and vitamin $\mathrm{E}$ on the lipid peroxidation, gamma-amino butyric and serotonin level in the brain of mice (Mus musculus) acutely intoxicated with lindane. J Neurol Sci. 2009;276:99-102. 